\title{
PERCEVAL Y LAS GOTAS DE SANGRE EN LA NIEVE
}

\author{
ducla el Amor cuál miís sul color sea, \\ - puirpura nevada o nieve roja. \\ (Don luis ne: Góncoks, Polifemo, \\ 107-108.)
}

Perceval, profundamente absorto en la contemplación we tres gotas de sangre en la nieve, colores que al mezclarse le rememoran el tinte de la faz de Blancheflor, su hermosa amiga, cleja una impresión perdurable en el ánimo de todo lector de $I_{i}$ contes del Graal '. El poético ensimismamiento sorprende y tal vez extraña cuando no se ha penetrado todavía en el seutido y el simbolismo de la última gran novela de Chrétien de Troyes. Su héroc, in. genuo y semisalvaje. hace poco que salió de la yerma floresta solitaria (la gostr foresl soutcine) impelido por la fuceza de la sangre. Rudo e incivil. sin conocer las más elementales reglas de la cortesía. había irrumpido en la suntuosa y relinada corte del rey Artús, pidiéndole que 'e

1 Li contes del (iraal. obra que Chrétien de 'Troyes dejó inacabada al morir, se fecha entre 1180 y 1191 . Es algo arriesgado ceñir mis la fecha; para Rru l.tjruswi, I. date du Conte du Graal de Chrétien de Troyes, Le Moyeen Age, IX, 1954, págs. 51-79, fué escrito e: 1180 o principios de 1181; para $\Lambda$. Foukk1E,, Remarques sur la date du Conte del (iraal de Chrétien de Troyes, Bulletin Bibliographique de la Socicte Intermationale Arthericnne, VII, 1955, prigimas 89-101, es postcrior al it dic mayo de 1181. 
hiciera caballero. En la corte reinala la desolación, ya fue. pooc antes. el Vermauz Chevalicrs de la Forest de Quinquerroi halsía contalo insolentemente en la sala real. se habia apoderado de la copa de Artús y había derramado su contenido sobre la reina, al propio ticmpo yue habia conminaldo a aquél a que se confesara su vasallo en caso de que ninguno de sus caballeros osara disputarle la copa, con ba cual se habia ido arrogantemente. El muchacho galis -que ni el lector ni il mismo saben todavía (pue se llama Perceval- es víctima de un cruel sarcasmo del scnescal Keus: si quiere armas, que vaya a quitarle las suyas al Vermatu Chevaliers. bi rey reprende al scnescal por burlarse de un muchacho ingenuo, y micntras tinto se suceden acontecimicntos ripides e inesperados. lil joven galess sin atender mucho a las razones del monarca. ha saludado a ma doncella de la corte, ha cual se ha echado a reir y ha dicho: "Muchacho, si vives mucho tiempo. mi corazón me asegura que en todo el mundo no habrá ni se conocerí mejor caballero que tú." Aquella doncella hacía más de seis años que no había reído, y ahora lo había hecho en presencia de todos. Keus, irrita. do. va hacia clla y le da un bofetón tan fucrte que la derriba por el suclo. y a continuación lanza al fucgo ardiente de la chimenea a un bufón de la corte que solía decir: "Esta doncella no reirá hasta que venga acjuel que tendrá todo el señorío de la caballería." Micntras el bufón grita y la doncella llora, el muchacho galés sale del castillo, va al encuentro del Vermauz. Chevaliers, lo vence, se apodera de sus armas y envía a la corte. por medio del escudero Yonet, la copa que fué robada al rey Artús. Yonet cumple el encargo y hace saber a la doncella que fuć abofeteada por el senescal que el muchacho ha prometido rengarla. lil butón, al oírlo, exclama gritando que ya se accrcan las venturas y que Keus scrá castigado antes de cuarenta días. pues el muchacho le romperá el brazo derecho. Este aticinio nos interesa particularmente, porque 
hat de cumplirse en el episodio de las gotals de sangre en la nieve que aquí se va a estudiar.

El inuchacho, con las arinas lermejas del caballero de la copa, llega al castillo de Gornemant de Goort, quien lo. arma caballero y le da lecciones de cortesía, cle prudencia y de manejo de las armas. Es recibido luego en la ciudad-castillo de Belrepeire, donde la señora, Blancheflor, doncella hermosísima, le pide que la auxilic en su crítica situación. La ciudad está sitiada por los ejércitos de Anguinguerón. senescal del rey Clamadeu cles Isles, y no. puede resistir ni un día más. El muchacho los vence a ambos en combate singular y los cnvía a la corte de Artús, con el especial encargo de anunciar a la doncella que fu: abofeteacla por Keus yue pronto serái vengada. Mientras tanto un profundo atmor ha nacido entre Blancheflor $y$ el muchacho, ef ctal parte de Belrepeire dispuesto a volver pronto y casirise con clla.

Sigue el fundamental episodio de la corte del Rico Rey Pescador, con el cortejo de la lanza que sangra y el graal. El muchacho, con su silencio, yerra y no concluye la alta empresa para la que estaba predestinado. Su prima, a la. que encuentra poco después, le hace saber cuán grand: ha sido su error y la magnitud del daño que su silencioha causado. En acjuel momento el muchacho "adivina" su nombre: Perceval. Sigue a este encuentro el del Orguelleus de la Lande, que es fácilmente rencido y enviado a la corte de Artús con el acostumbrado encargo para. l. doncella abofeteada por Keus. El bufón vuclve a anunciar que pronto recibirá el senescal el castigo que merece..

He resumido rapidísimamente algunos de los trancesde la novela que preceden al episodio de las gotas de sangre en la nieve, que se desarrolla del modo siguiente":

2 Versos 4.141-4.602 de la edición de M.fon's Hil.ka, Der Perceialroman (Li conces del Graal), ron Christian von 'rroyes, vol. V de. "Christion von Troyes siimtliche crhaltene Werke", Halle, 1932. Tod3s. lasi citas y referencias que hago a $L i$ confes del (iraal se basan en. esta clición. Mlientras corrijo estas pruebas me llega una nueva y cx-. 
Fi rey Ariús moviliza su corte para ir en busca del muchacho, de quien tantas proceas llegan a sus oídos de boca de adguellos que ha vencido. Con lucido acompañamicnto sale de Carlion y por la noche acampa en un prado, al lado de un bosque. Al amanecer, cstando todo cubierto de nicve, Perceval. en busca de arenturas, llega a aqual prado $y$, sin advertir las tiendas del campamento real. ve una manada de ánades perseguida por un halcón el cual se precipita sobre uno de cllos y le hiere en el cucllo; pero el ave puede escapar y remonta el vuelo, dejando tres gotas de sangre sobre la nieve. Perceval con. templa las tres gotas, cuyo color. mezclado con la blancura de la nieve, le sugiere la fay de su amiga Blancheflor. y quedia alssorto mirando las manchas deste el caballo. Asi estuvo largo tiempo, ensimismado, hasta que fué visto por les escuderos que se levantaban y salian de ias tiendas reales. Sagremor le Desreci va a la tienda de Artús, que aun dormía. y le notifica que en el prado hay un caballero desconocido: y el rey le ordena que vaya en su busca y lo traiga a su presencia. Sagremor se arma, monta y va hacia Perceval, al cual, con imperiosas palabras. conmina para que se presente ante el rey. Al no responderle ni hacer ningún caso de el, Sagremor lo ata. ca, y Perceral, al darse cuenta de ello, lo acomete a su ve\% con la lanza y lo derriba. Ll caballo del vencido llega a las tiendas. lo que provoca los sarcasinos del senescal Keus, que se burla de Sagremor; pero el rey le reprende y le ordenal que vaya él en busca del desconocido. El senescal se arma y a caballo se encamina hacia Perceval, que nuevamente estaba cnsimismado en la contemplación

celente ediciún de Li contes del Graal, preparada por el profesor Wiuhnar Roach, de l'cnnsylvania: Chrcitien de Troyes, Le roman de Perceval ou Le conte du Graal, colección "Textes littéraires français", ed. Droz-Giard, Gincbra-Lille, 1956. Da el texto del manuscrito $\mathbf{T}$ (fr. 12576 de la Bibl. Nat. de larís) Y conserva la numeración de rersos de la de Hilka, lo que se acuerda con las citas que hago en el presente articulo. 
de lass getats de sangre. Desabridamente le dice que vayat a! rey y lo atalea, pero perceval con la latmza lo hace caer sobre una piedra y se rompe el brazo derecho entre el codo y la axila, tal como el bufón había vaticinado. Ed caballo regresa a las tiendas, los de la corte recogen a Keus desmayado y Perceval sigue entregado a su contemplación. Gauvain dice al rey que no es justo apartar a nin. gún caballero de sus pensamientos, que han obrado mal Sagremor y Keus y que él está dispuesto a ir al desconocido y a traerlo al campamento amistosa y cortésmente. Con licencia del rey, Gauvain sc aproxima a Perceval. Las gotas de sangre ya estaban casi fundidas en la nieve, y poco le costio, con amabilidad y comprensión, lograr que departicra con él. Perceral le cuenta lo que había estado contemplando y que lass gotas de sangre le hacian pensate en el rostro de su amiga. Gauvain, que adinira la "cortesia" del ensimismamiento, le explica que a quien ha roto el brazo es a Keus. y Perceval afirma que ya está vengada la doncella que por acuél fuć abofeteada. Gauvain comprende entonces que aquél es el caballero que el rey va buscando, y amistosamente llegan ante Artús, que recibe afablemente a Perceval, le pide que jamás se aparte de su corte y hace patente que se ha cumpliclo lo vaticinado por el bufón. Al llegar la reina, Perceval la saluda con gran cortesía y luego besa y abraza a la doncella que no. rió hasta verle.

Dentro de la trama de Li conles del Graal el episodio de las gotas de sangre en la nieve tiene una función clara y es fundamentalmente necesario. En primer lugar, acerca Perceval al rey Artús, que tiene vivo empeño en incorporar a su corte a aquel extraordinario caballero que constantemente de envia a los que ha vencido. Pero mu. cha mayor importancia tiene que el epjodio motive un combate entre Perceral y el senescal Keus. en el cual aquid quichra el brazo derecho de éste y así se cumpla el vati- 
cinio del butón y cllo haga ciettas las palabras de la doncella abofeteida. Recordenues que ésta. al ver por vez: prinera al muchacho galés en la corte. echó a rér y dijo:

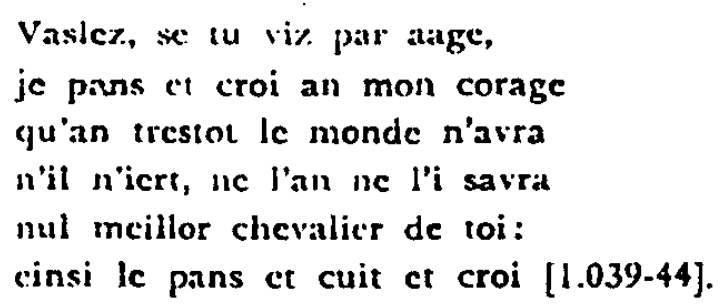

[Nuchacho, si te clurat la vida, mi corazón me dice que en todo. (-) mundo no habrá, ni se cncontrara ni se conocerá ningún caballero. mejor que tis: asi lo picnso, lo supongo y lo creo.]

Si el senescal Keus aboletea at la doncella y lanza al fuego de la chimenea al bufón es porque éste, como sin. duda saben codes los de la corte,

soloit dire:

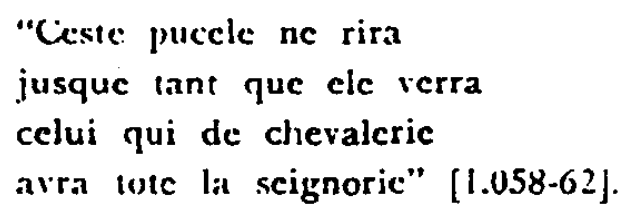

[... solía decir: "lista doncella no reirá hasta que rea a aquel queicndrá todo el señorio de la cabalicría".]

Cuando el esculero Yonct regresa a la corte con la copa y explica la primcra victoria del muchacho, el bufón comprometc la veracidad del vaticinio de la doncella cons otro más arricsgado por lo concreto y próximo:

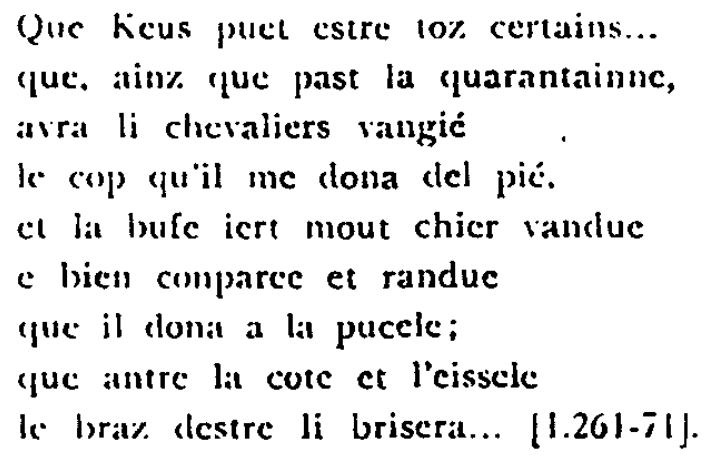


[Keus puede estar bien seguro... que, antes de que pase la cuarentena, el caballero habri vengado el puntapié que tne díb y será .pagado caro y bien devuclto el bofetón que dió a la doncella; pues le romperá el brazo derecho entre el codo y la axila.]

Este segundo vaticinio se cumple al pie de la letra en inuestro episodio:

\author{
Eit Percevaus... \\ desor la bocle an haut l'ataint, \\ si l'abati sor une roche \\ que la chanole li esloche \\ ct qu'antre le code et l'eissele \\ ausi come une seche estele \\ l'os del braz destre !i brisa, \\ si con li fos le devisa \\ qui maintes foiz deviné l'ot: \\ voirs fu li devinaus au sot $[4.307-16]$.
}

IY Perceval le dió cucima de la bocla de modo que lo derribó sobre una roca de sucrte que le dislocó la clavícula y entre el codo $\checkmark$ la axila, como si fuera una astilla seca, le quebró el hueso del brazo derecho, asf como habla anunciado el bufón, que muchas reces 'lo habia predicho. Verdadero fué el vaticinio del tonto.]

Desde este momento. y bien lo subraya Chrétien de Troyes, tanto los lectores de la novela como los caballeros y las damas de la corte de Artús saben que Perceval de chevalerie aura tote seignorie. La concatenación de vaticinios se ha hecho cierta precisamente en nuestro episodio, en el cual el novelista ha conseguido que existiera una razón para que Perceval, caballero en realidad ads: crito a la corte de Artús, combaticra con Keus, senescal del rey.

La estructura del cpisodio se halla, de un modo casi idéntico, en otro del Erec et Enide, primera novela de Chrétien de Troyes. El joven matrimonio Erec y Enide. que hace tiempo que vaga en busca de aventuras, llega a una llanura próxima a un bosque en el cual aquel día ha. bia acampado y montado sus tiendas y pabellones el rey Artús, acompañado de la reina y de los barones de su 
corte, dispuestos a dedicar unas jornadas a la caza. El senescal Keus se aparta del campamento. val al encuentro de Erec. sin reconocerlo. le pregunta quien es y le indicat que vaya a reunirse con Artús. Al negarse a ello Erec, insiste con insolencia, llegan a las armas y el senescal recibe tal golpe en el escudo. que éste le da en la sien y'e aprisiona el brazo contra el pecho, y cac tendido en el suelo. Cuando Artús se entera del incidente ordena a su sobrino Gaurain. "que siempre fue franco y cortés", que sc aproxime al desconocido y "amablemente". le pregunte quién es y procurc llevarlo a su presencia. Gauvain se acerca a Erece lo saluda cortésmente y con palabras afables y comedidas lo entretiene hasta que el campamento real se trasladia a clonde se hallan los dos caballeros y Enide, y lirec, linalmente. se da a conocer y accede a reunirse con Artis, la reina y los barones".

El parcido entre ambos episodios es patente, aunque en $L i$ conte's del Gral intervenga Sagremor. que falta en el Erec et Enide. Tanto Erec como Perceral se encuentran próximos al campamento de Artús. de cuya corte hacía tiempo que se hallaban ausentes; Keus es descomedido e insolente con ambos, y de ambos recibe un golpe que lo derriba y lo castiga; la gestión afable de Gauvain es lo que lleva al caballero junto al rey y su mesnada. Es perfectamente lícito concluir que Chrétien de Troyes, al escribir el episodio de Li conles del Graal, tuvo muy en cuenta el del Erece et Enide.

Como es natural, el gran poeta que fuc Wolfram von Eschenbach no pudo menospreciar el bellísimo episodio de las gotas de sangre en la nieve. Lo recoge, adapta, am. plifica y varía en algún aspecto en el libro VI cle su Par-

3 Versos 3.907-4.118 de la cdición de Mario Roques, Les romans dr Chréticn de Troyes. I, Erec el Enide, "Les classiquucs français du Noien Age", Paris, 1953. 
zival. Lil escritor baivaro situia la escenal al orillas del rio Plimizoxl, donde Artus habial establecido su campamen16 para entregarse a lat cazal de halconcria. Uno de los mejores halcones del rey habia huido y habia pasado la noche en un boscfue proximo. donde también se encontraba Parzival. Al amamecer, Parzival contempla cómo el haicón persigue una bandacla de más de un millar de ánades. ataca a uno de ellos y la nicve queda manchada con tres gotas de su sangre. Al rerlas Paryival, "que tenía el corazón fiel”, sc. dice a sí mismo:

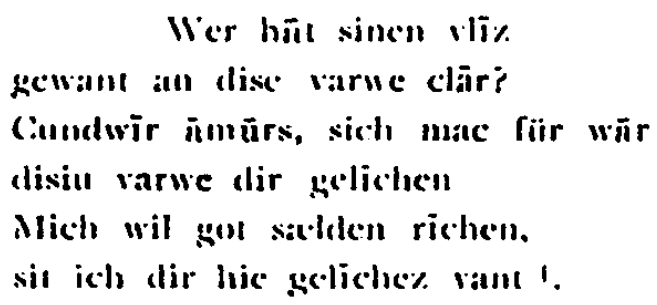

Qui done a pris le soin de latire apparaitre ici es fratiches couleurs: Condwiramour, en vérité, c'est at ton visage qu'il faut les comparer. Dieu a voulu me combler de joie en me faisant voir ici l'image de ta beauté 5. .]

Ei soliloquio es muy explicito: Parzival re en la mezclat de la sangre y la nieve la imagen del hermoso cuerpo de C.unclwiramurs. nombre que el adaptador alemán da ir Blancheflor. Pero ororgat a la imagen unas caracteristicas yue no se hallan en Chrétien:

$$
\begin{aligned}
& \text { Des heldes ougen märin, } \\
& \text { als c\% dort wats crgangen, } \\
& \text { awēn zaher an ir wangen, } \\
& \text { den dritten an ir kinne is. }
\end{aligned}
$$

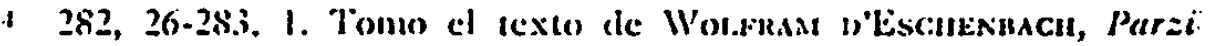

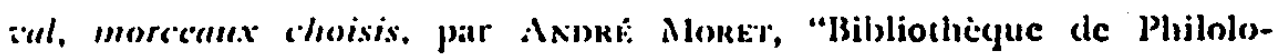
gic Germanique”, l'aris. 194.i, paig. 128.

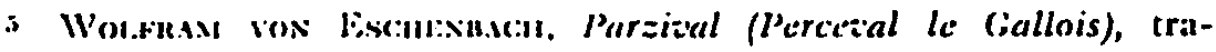
ducción francesal de Ernest Tomnclat, l'aris, 193t tomo I, paig. $2+6$ i. 28.3, $10-1.3$. 


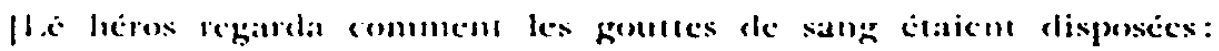

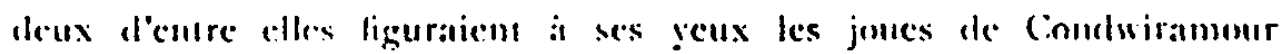
al le trnisicme son menton'.

l'aryival se chsimismal en csta contemplacion. ansente de cuanto ke rorka. inmóvil hasta el pumto que se le creeria dormido. Un paje advierte a los del campamento de

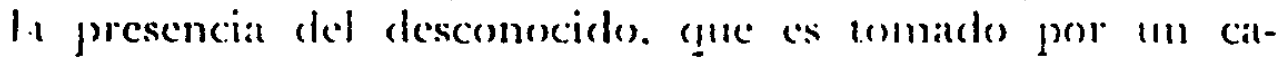
ballero enemigo. Segramors. con la interessión de la reina. obtienc que Artús le permita ir hacia acped caballero. y con poco esfuerzo por parte de Parzival es derribado. A continuación intenta la empresa el senescal Keic: y antes de narrar el combate Wolfaum hace ma insocación a! Amor ffrou Mimme). y' aluele al poesa lleinrich ron Vel-

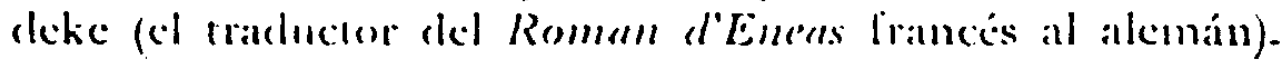
Keic es derribado por Parcival. y al caer se quielora una pierna y el brazo derecho. Gawan. sin armas. se acercal a l'arzival. que estaba tan abstraido en ba contemplación de las gotas de sangre que ni tim solo reparó en su presencia. Entonces Gawan cubrio las manchas de sangre con un velo de scela de Siria. con lo que l'arzival salio de su ensimismamiento. se hizo asequible a la comversación y se incorporó al campancuto de Artús.

bi episodio de las gotas de sangre en la nieve no reapa. rece en las numerosas continuaciones e imitaciones de $L$; conles del Granl ni en los clemás rommos de la materia de Bretaña. Solamente en el Trislin francés en prosa halla. mos una imitacion del episodio, de la cual también Percoval es el protagonista. Véase. segúm el resumen de I,̈̈seth :

Un jour dhiver. Perceral passe derant la cour de Carlion. Il roit. sill lat neige. trois guentes de sang, qui le fout penser à Helaine fhromaner de comiain) sans pair, une belle dame de lat cour. et se perd dans une rêserie contemplative. A la cour, on croit que éest "11 cheralier qui veu jouter: Keu. Mlorderer. Gauvain fo Agreatain| se presentent et sont successivement désarçomés; le vainiqueur s'éloigne.

; Tratc. Tonnclial, I, pig. 2t6. 
Line demoiselle ctrangère. venue ce jour-là à la cour, fait enfin-savoir sue c'est l'ercetall le Gallois *

lis necesario, linalmente, considerar con detención la versión del episodio de Perceval y las gotas de sangre en la nieve en el relato galís. impropiamente llamado mabinogi, que lleva el título de Peredur ab Evrirece, texto sobre el que todo el mundo parece de acuerdo en adnitir que es posterior a $L i$ conles del Graal y que procede de una inmediata fucnte francesa. No es ahora la ocasión apropiada para debatir sobrc la relación existente entre Li contes del Gronl y el Peredur; y ameque, sin duda, lo más prudente e's aceptar que el autor del relato galés tuvo muy presente la obra francesa, cuyo profundo sentido no entendió y cuya trama destrozó lamentablemente. no se. ría licito descchar la opinión defendida por el sector celtizante de la crudición artúrica. l’ara Miss Mary Williams ". R. S. Loomis "" y otros, el Peredur deriva de un relato anterior a Chritien de Troyes; W. A. Nitze" sostiene que el Peredur indudablemente manifiesta influjo de Chrétien de Troyes; Jean Marx hace notar que "le personnage de Peredur, le héros central du récit, a évidemment subi linfluence du l'erceval français et à peu près sûrement celle de Chritien de Troyes avec le texte duquel certains passages offrent des ressemblances saisisantes" $1:$.

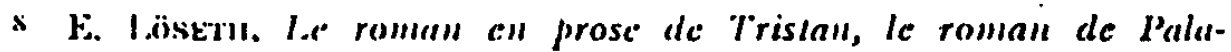
méde at la compilation de Rusticicn de Pisc. Analyse critique d'après Ja:: manuscrits de l'aris, Paris, 1891, "Bibliothicque de l'Ecole des Hautes litudes", paig. 2tt, \$ $\mathbf{3 1 3 .}$

" M.nx W'nlinus, Essui sur la composition du roman gallois de Peredur, l'aris, 1909.

1" Rogre Sulmmax Looms, Arthurian tradition and Chréticn de Troyes, New York, 1949, pig. 37.

11 Whis.t.M A. Nitz.r., Perceiul and the Holy Grail: An Essay on the Rumance of Chréticn de Troyes, "University of California Publications in Mlocicn Philology", XXVIII, 5, 1949, pig. 311.

1. Juix Mlıkx, La légende arthurieme et le Craal, Paris, 1952, página 383. 
Véase de qué modo el episodio de Chrétien de Troyes aparece resumido cn el l'eredur, según la versión francesal de J. Loth:

V'ers le soir, il atriva dans une vallec, et, au bout de la vallee, devant la cellule d'un serviteur de Dicu. L'ermite laccucillit bien, et il y passa la nuit. I.c lendemain matin, il se leva et sortit. Il etait tombe de lat neige penclant la nuit, et un faucon avait tue un canard devant la cellule. Ic bruit du cheval fit fuir ke faucon, et un corbeas s'absuttit sur la chair de l'oiseall. Percelur s'arréla, et, en royant la noirceur du corleall, la blanchcur de la ncige, la rougeur du sang, il songea ì la chevelure de la femme qu'il ainatit le plus, aussi noire que le corbeau un le jais, it sa pcau aussi blanchc quc la ncige, aux pommettes de ses joues, aussi rouges que le sang sur la neige. Or, it ce moment, Arthur el sit cour étaient en quête le peredur... 1.3.

Y sigue el episodio de acuerdo con el texto de Chrétien: un paje (no Sagremor) ataca a Percelur y es derribado: Kei corre la misma suerte y se rompe el brazo y el omóplato. y' (iwalchmei (o sea, Galurain) sc acerca cor. tísmente al caballero. departe con él y logra llevarlo al campannento. Peredur ha explicado a Gwalchmei la calusa de su ensimismamiento con las siguicntes palabras:

... je méditais sur la lemme que j'aime le plus. Voici comment son sourenir mest renu. En consiclérant la neige, le corbeau et les taches de sang du canard tué par le faucon sur la neige, je ne mis ¿ penser yue sa peau ressemblait à la neige, la noirceur de ses chereux et de ses sourcils au plumage du corbeau, et les deux pomneleties de ses joues aux deux gouttes de sang ${ }^{14}$.

Ia trama del episodio responde exactamente a la de Li conles del Grent. pero el tema preciso de la nieve y la sangre ofrece ciertas diferencias esenciales que llaman la atención.

13 Les Mabinogion du Lizre Rouge de Hergest aiec les iarianles dil Liere Blanc de Rhyelderch, iraduit du gallois... par J. I.om, II, laris, 1913, paigs. i6 y 77. Version igual en la traducción inglesa The Mabinogion translated by Gixix Jonks and litoms Josrs, "Everymav's Library", londres, 1949, piig. 199.

in Trad. Lotll, ibid., paig. 80; cfr. trad. Joxws, ibid., pig. 201. 
Precisamente, en lo que difieren los textos de Li contes del Graal y del Percedur se ha visto, desde hace tiempo. un argumento a favor del origen céltico de los temas literatios desarrollados en las novelas de la materia de Bretañal 'i. Lin efecto. en 131 poema irlandés titulado $E /$ desticroro de los hijos de Usnech, "dont te manuscrit plus ancien parait antéricur a $1164 " 1 "$, se narra lo siguiente: Lin cierta ocasión. el padre adoptivo de la hermosa doncella Deirdre estaba desollando un ternero, a la intemperic y sobre la nieve, para dárselo a comer. Deirctre obser vó que un cucrvo bebía la sangre que había caído sobre la nice y dijo a su aya Leborcham: "Digno de amarse seria el hombre que reuniera los tres colores que veo alli: cl cabello como el cuervo. las mejillas como la sangre y el cuerpo como la nieve." A lo que I,eborcham replicó que quien reunia lakes condiciones no se encontraba lejos de alli: era Naisi. hijo de Usnech '".

R. S. Loomis, ante estas tres versiones (la irlandesa de El desticros de los hijos de Usnech, la francesa de Li conses del Granl y la galesa del Peredur). concluye que el rema irlandis se ha manteniclo en su integridad en el Peredur. y que Chrétien. "o su fuente", ha eliminado el cucroo por la sencilla ràón de yue los franceses no gus-

1.) lat relacioin entre el pocma irlandés y este episodio de los tex-

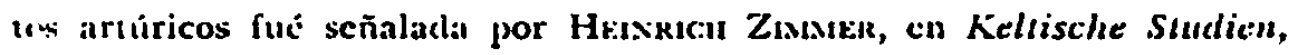
1!. Berlin. IsSt. piiss. 201 y sigs. Vénse la bibliografia posterior y una exposicion favorable a la iendencia celtizante en el curso de Jis.

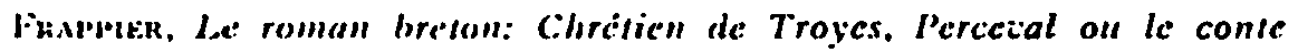
dh. Ciraul. Paris. 1953. pigs. 68.71, en las publicaciones en ciclostilo "les cours de la Surbonne".

16. J. lam. o. c., 11, piig. 76. nola 3.

1; Doy el resumen del episodio segin la versión que ofrecen 11. S. L oomis, en Arthurien tredition, pigs. $+1+y+15$, y W. A. Nitz:, en Percrial and the Holy (iruil, paig. 311. Del Desticrro de los hijos de lishech existe ataptacion espaniola de algunos cpisodios, a través de traducciones inglesas. en la antologia l.a pocsia irlandesa, version, selección y proilngo de M. Mlıxtir, Barcelona. 1952, págs. 34-37, colecirin "W Mensaje". 
tabaun de las mujeres morenas, sino de las rubias, y asi Blancheflor tenía los cabellos, que parecian "de fino oro" 18 Nitze. por su parte. que tan brillantes contribucioncs ha hecho en pro de la lesis céltica. llega a la conclusión de yue ni el l'ercelar ni hi eomles del Cormal toman la comparación de El desticreo de los hijos de Usnech. sino-de alguna version pojular transmitida en forma oral "'. Creo que la solución de Nitze es la acertada. aumque el problema es, sin duda alguna. inucho más complejo. y el tema. si bien ofrece una rayectoria y unas características típicannente radicionales. aparece en Chretien de Troyes pro. fumbamente contaminado con la literatura culta.

lil eprosolio que aqui consideramos, descle que el cabatHero advierte las getats de sangre en la nieve hasta que. por fin, se incorpora al campamento del rey drtús es, cn colos sus dealalles, y en la letra de la mayoria de sus pa. - sajes. exalcto en t,i comles del Graal y en el Peredur. Para este cpisodio no es necesario recurrir a la existencia de ma hipotetica luente común. tau tenazmente defendida por un sector cle la crítical celtizante. A cualquiera que, libre de prejuiciess. y sabiendo yuc la novela francesa es cronológicamente anterior al relato galés. considerc ambos textos, $k$ es evidente que Chrétien de Troyes es, en este caso preciso. la luente del mabinogi. La única discrepancia se halla precisanente en los elementos que in. terviencn en la compatación entre el fenóneno natural y los colores de la mujer. I o más notable de esta discrepancia es. como ya hemos visto, que a la impresión de blancura de la nieve y de rojez de la sangre el Peredur añade la de la negrurar. sugerida por el plumaje del cuer. ro. I siendo así (fuc esta adición tambien se halla en $E l$ destierro de los hijos de Usnech, se ha creido que el Pe-

is Artherian tradition. prig. +15 .

1.' Percesal and the Holy Grail, pig. 312. 
redur mantenía en su integridad un viejo tema irlandés que Chrétien redujo y desfiguró.

Es preciso enfocar el problema desde otro punto de vista. Nadic ignora que existe un muy difundido tema folklórico que en el Motif-Index, de Stith Thompson ${ }^{20}$ se cataloga en los apartados T 11, 6 (IVish for wife red as blood, white as snow, black as raven) y Z 65, 1 (Red as blood, white as snow... sometimes: black as a raven). Su difusión en la literatura tradicional se debe principalmen. te al hecho de constituir el primer motivo de numerosas versiones del cuento de Blancanieves. .En 1913, J. Boltey G. Polívka registraban este cuento en el folklore fla. menco. danés, succo. noruego, islandés, inglés, escocés, francés. italiano. catalán, portugués, vasco, gricgo, albanés. rumano. servo-croata, búlgaro, checo, eslovaco. wendo, polonés, ruso. lituano. letón, finlandés, húngaro, turco e indio". Basta esta enumeración de culturas, tan distintas y tan alejadas. para poner de manifiesto que el cuento ha de ser muy antiguo.

Reparcmos en algunas. muy pocas, versiones de este tema que pueden ayudarnos en nucstra indagación. Fir Lo cunto de li cunti o Pcutamerone, de Gianbattista Basile, famosa colección de cuentos en dialecto napolitano. que se publicó en $163+$ y 1636 . encontramos dos curiosas versiones del motivo. en las cuales la nieve, sin duda algo. exótica para estos relatos meridionales, ha sido sustituída por otro elemento blanco. En la novela Le tre cetre (IX de la jornada V). un príncipe se corta en la mesa,

20 Motif-Index of Folk-literature, by Snmi Tilostpson, Helsinki, 1932-36, "FF Comunications", núms. 106-109 y 116-117.

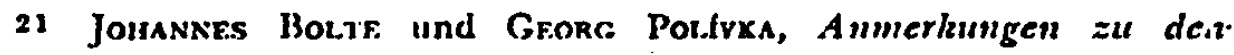
Kinder- und Hausmärchen der Brïder Grimm, I, Ieipzig, 1913, pá ginas 453-46t. Véase también ANrit Aark: y Strtu Tuompson, The Types of the Folk-tale, a classification and bibliography, Helsinki, 1928, "FF Comunications", Mim. 74, tipo 709; y para las versionesespañolas, Ralph S. Bocos, Index of Spanish Folkıales, Helsinki, 1930, “FF Conunications", mun. 90, tipo 709. 
calen dos gotas de sangre cncima del requesón y decide buscar una mujer que réuna aquellos colores:

... occoryc che trovalose no juorno tutte neicmme a tlavola, vo kemos lo Prencepe tagliare na recotta pe mmiezo, mentre tenera mente a le cciarole che passarano, se fece desgraziatamente no ntacco 1 In dito tale che callenno duje stizze de sango neoppa a la recotta, feccro na inesca de colore accosi bello e graziuso che, o fossc castico. d'Ammorc, che l'aspettara a lo passo, o volontit de in Cíclo pe ccon. a)lare chillo omino dia bene de lo Patre, non era tanto molestato da la. polletra domestaca quanto da sto pollitro farrateco era tomentato, le: renuc capriccio de trevarc na fenmena accossi ghianca c rrossa: comme cra appunto chella recotta tenta da lo sango sujo 22.

Lin la novela Lo cuoreo (IX de la jomada IV). Basilenarra que el rey Milluccio halló cazando un cucrvo mucrto sobre una piedra de mármol, y la contemplación de los tres colores de hio descar una esposa blanca y roja, como. la piedra. y de cabellos negros, como el phumaje del cuervo:

... Mlilluccio, to Rre de Fratta-ombrosat, lo quale cra accossi perduto pe la caccia... che no journo lo portaje la Fortuna a no vosco... dose 'ncoppa na bellissema preta matrnora trosaje no cuorvo che frisco frisco era stalle acciso. Io Rre veclenno chillo sango vivo vivo' sghizeiato sopra chella preta janca janca, jetlanuo no gran sospiro, disse: "() ciclo, e non porria avera na moglicre accossi ghianca e rrossa comme achella preta, e che aresse li capille c le cciglia accossi negre comme so le ppene die chisto cuorvo?" 2.3

Ia vimos que. en El desticro de los hijos de Usnech, la doncella Deirclac contemplaba la sangre de un ternero. que cstaba desollando su padre adoptivo, sangre que caía sobre la nieve y que cra bebida por el cucrvo. El cucrvo: nos ha aparccido en el cuento napolitano de Basile; la sangre de una res aparece en un cuento extremeño titu.

22 Tomo el texto de la colicion Il Pentamernnc del Caualier Gio. zan Batrista Basile, ovicro Lo cmino de li cumle, tomo II, Nápoles, 1788, prigs. 196 y 197. Sobrc esta obra de Basile, véase B. Croce, Saggri sulla letteratura ituliana del seicento, Bari, 1911, pigs. 1-122, y Storia della elì barocca, Bari, 1929, págs. 445-466.

2.3 lbid., pig. $\mathscr{Y}$. 
lado El rey durmiente en su lecho, recogido en $1886^{\circ}$ por I-Icrnández. Soto:

... Un dia que habia caicle una gran nevacia, todo el campo estabia tan blanco que daba gusio verlo. Se asomó la princesa a un bal. cion a ticmpo que un pastor estalsa degollando un borreguito, y lat sangre caia en el suclo manchanclo la nieve. Un zagal que estaba mirando la facena se quecló mirando el contraste que hacía el color cle la sangre con la blancura de la nieve $=1$.

I al situación descrita en este chento extremeño es similar a la que hallamos en el catalán titulado Sang-i-neu, recogiclo en 1885 por Maspons y Labrós:

Bra una regacla un princep que s'estava un dia en una finestra si: son palau miram com queya una gran nevada; héuse aquí que jugant ab un ganivetet que tenia cn les mans, s'feu un tall y caygué una golat de: sameh damunt lat nell. Fill que la vell y cliu: "No'm vull (alsior sino al) quil's diga sanch-y-neu" 25.

Como puede rerse, el elemento negro, por lo general sugerido por el plumaje de un cuervo, pucde desaparceer en muchas versiones. Io permanente es la blancura (sugericla casi siempre por la nieve) y la roje\% (sugerida siempre por la singre). Los hermanos Grimm nos ofrecen las slos variantes. En el cuento El enebro (Van den Machandelboom, núm. ti de la edición de 1812) leemos:

... Frence a su casio, en un patio, crecia un enebro, y un dia de imierno en yue la mujer se hallaba debajo de él mondando una manzana. cortóse en un dedo Y la sangre cayó en la nieve. "IAy! -exclamó con un profundo suspiro. y, al mirar la sangre, le entró una gran melancolia--: iSi luvicse un hijo rojo como la sangre y blanco como la nieve!" 2ni

\#1 Cirentos popmlares de Extremudura, recogiclos $y$ anotados por S. Hirkinidiz Sorn. I. prig. 106. en "Biblioteca de las tradiciones pojulares españolas". X, Marlrid, 1866.

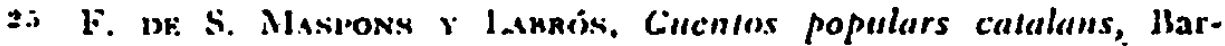
colona. 1885, pizg. 18.

in Cimentos completos de los llermanos cirimm, traducción di- 
Iin el cuento de Blancanieves (Snecwillchen, núm. 53 de la edición de 1812) aparecen los tres colores. pero el negro. que un ligura como sugerido por el cucrvo, queda un poco postizo:

Lara un crute dia de invictuo, y los copess de nieve catian del ciclo como blancas plumas. lat reina cosia jumo a una ventana, cuyo marco era de chano. I como mienuras cosia miralba cacr los copos, com la aguja se pinclori un dedo, y tres gotas de sangre fueron a eacr sobre: la nicte. bi rojo de la sangre elestacabal hellanente sobre =l fonelo blanco, ! ella pensi: " $j \lambda$, si pudiese tener una hija que luese blanca como la nieve, roja como la sangre y negra como el ébano de esta rentana!" No mucho tiempo despues le nació una niña yue cra blanca como la nieve, sonrosada como la sangre y de cabello axegro como la marlera de ébano; y por cso le pusicron por nombre Mancanicres $2 i$.

Fácil seria multiplicar los cjemplos, de las más diversist procedencias. de versiones con tres colores (blanco, rojo y negro) y versiones con sólo dos (blanco y rojo). Insisto en este aspecto para poner de relieve que Chrétien de Troyes pudo muy bien conocer alguna de las versiones del morivo a base de dos colores, que cs la que aparece en $L i$ iontes del (irual, o. conociendo las de tres, suprimir el negro. El redactor del Peredur, por su parte. pudo muy bien ir siguiendo el texto francés de Chritien, y. al llegar a este motivo y recordar las versiones de tres colores. añadir el clemento cuervo-cabellos negros por su cuenta.

Ni Sagremor le Desrec -o sea. "el atulondrado"-, ni el malhumorado y tosco Keus. eran capaces de comprender el ensimismamiento de Pcreval. Gauvain. sí. el procotipo de la mis refinada cortesía. experto en armas y en anores, aungue en éstos con donjuanesca volubilidad. Pro. testa de la actiud de los dos primeros, porque

recta del alemín por likasciscon Payakots, revision y prólogo por

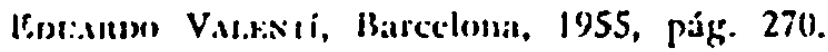

25 Ihidl., piig. 526. 
... cheraliers autre ne doit oster, si con cil clui ont fet, cle son panser, quel que il l'et...

l.i chevaliers cl'aucune perte estoit pansis qu'il aroit feite, ou s'amic li crt forstrcitc... [\$.35+-62].

[... ningún caballero debe apartar a otro de sus reflexiones, seanlas que fueren, como han hecho aquelios dos... El caballero (Perceval) estaba pensativo por alguna pérclicla que habla sufrido o porquesil amiga le habia sido robikla...]

Gauvain sale que quien está albsorto y sumiclo en sus. reflexiones demuestra cicrto refinamiento espiritual, y su intuición le hace adivinar que el ensimismamiento de l'erceval es amoroso (oul s'amie li erl forstrcile), pues, como. muy bien señala Nitze ${ }^{2 *}$, todo el cpisodio tiene un senlido cortés y amoroso que parece ser una glosa de lo que Andrea Capellanus había escrito en su tratado $D c$ amore, donde, entre las regulue amoris, figura la siguiente: Verus amans assidua sine intcrmissione coamantis imaginationc detinctur"." I por esto, cuando ha logrado entablar conversación con Perceval y éste le ha explicado cual era la causa de su ensimismamiento, Gauvain ha comentado:

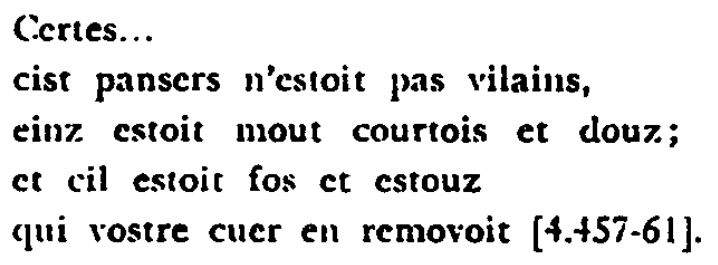

[Realmente, csta reflexión no cra villana, antes bien era muy cortés y dulce, y fueron necios y estúpidos los que apartaron rucstro corazón de ella.]

El pansers de Perceval no era vilains, sino courtois. $Y$ ello es de suma importancia en la evolución moral y psi-

:s W. A. Natz:, Perceval and lise lloly Grail, pág. 298.

29 Tomo la cita de Anbra Capkitrano, Trablato d'amore, ed. Sial. vatore Battaglia, Roma, 1947, pag. 359. 
cológica del protagonista de Li conles del Graal, novela en la que, sin duda con acierto, se ha visto una clara intención elucativa, "un roman éducatif en action", como ho dicho Alexandre Micha ${ }^{31 .}$ Eil joven semisalvaje del principio de la novcla. que entró por vez primera en la corte del rey Artus ignorando las más clcmentales nomas de la clucación y de la etiqueta, es ahora un caballero capaz de sumirse en un pouser courlois. Y ello cs tan cierto que, cuando (Baluain lo lleva al campamento y aparece la reina, Perceval la saluda con palabias que contrastan intencionadamente con la groscría de antes. Nos sorprende con esta cortesísima salutación:

\author{
Deus doint joic et enor \\ a la plus bele, a la meillor \\ de cotes les dames qui soient, \\ tcsmoing to\% les iauz qui lat voient \\ ct to\% (atus qui reiic l'ont! [4.587-91].
}

(1)ios de gn\%o y honor a la mis hermosa, a la mejor de todas las elamas que existen, como atestiguan los ojos que la ven y todos cuantos la han visto.!

Reto R. Beryola, que hat estudiado muy agudamente el sentido del episodio de las gotas de sangre en la nicve, comental las antes citadas palabras de Gauvain del siguicute modo: "Qu y avait-il de corlois $e$ dolz dans la rêveric de lerceval, yue cachait-elle de si grand que fols c estolz était celui qui l'en détournait sans comprendre sa valcur ni son sens? La pensée du beau visage de son amie évoqué par trois gouttes de sang dans la neige ne pouvaitelle venir à un amant quelconque, même à un "vilain"? Certes! mais un amant quelconque, un vilain, un Sagremor ou un $\mathrm{Keu}$, aurait difficilement passé toute la mati-

31) Alexandul: Mlicin, le: l'crceval de chréticn de Troyes (roman ćducatif), en Laumière du (iraal, "Cahicrs du Sud", 1951, pág. 130. El mismo aspecto cducativo se cstudia en el trabajo de Rita Lejeuni, La date du Conte du Graal de Chrétien de Troyes. Le Moyen Age, LX, 1954, págs. $51-79$. 
néc absorbe par celle pensice, il ne siy scrait gucte replonge apres avoir abatu deux adversaires. Gatuvain lui-même n'aurait guère ríussi à y arracher Perceval. si une force supéricure, le soleil, nérait intervenue alec cette même fatalité des événements qui avait produit les gouttes de sang dans la neige. en se servant des oies satuvages et du falcicon":"

Vamos dándonos cuenta de que en el episodio de $L i$ contes del Graul convergen una seric de intenciones del escritor. Ya vimos que era absolutamente necesario para que, provocando la lucha entre Perceval y Keus y el rompimiento del brazo de éste, quedara bien patente que el héroe de la novela estaba destinado a ser el mejor caballero del mundo. Ahoral advertimos que el ensimismamicn. 16) ante las gotas de sangre en la nieve es una clara prochat de que perceval, el predestinado, hat alcallyado ya el mayor grado de la cortesía. lil vicjo motivo folklórico se ha llenado de un sentido courlois, y, como vamos a ver pronto, se ha vinculado a un símil poético de origen culto y literario. Pero para llegar a esto último nos es preciso detenernos en lo más concreto del motivo, tal como aparece en Chrétien de Troyes.

Tenemos que precisar exactamente la imagen colorista que tanto impresionó a Perceval. El ánade fué herido en el cuello y derramó

$$
\begin{aligned}
& \text {... trois gotes de salnc, } \\
& \text { yui espandirent sor le blanc, } \\
& \text { si sanbla natural color [4.187-89]. }
\end{aligned}
$$

[... tres gotas de sangre que sc esparcieron sol)re lo blanco y pareció color natural.]

O) sea. que lo que sugiere a lereceval el recuerdo de blancheflor es que la me\%cla del rojo de la sangre con el blanco de la nieve producen el color somrosado de lar

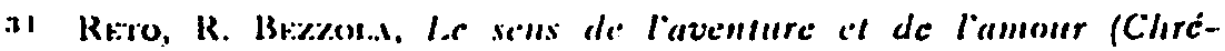
lien de Troyes), col. "I.a Jeune l'arque", l'aris, 194i, paig. 29. 
carnación humana: concretamente, el color del rostro. billo. queda corroborado en todos los pasajes en gue se hace mención del tenómeno:

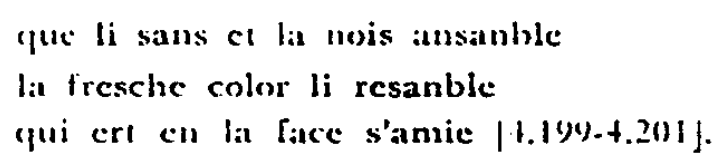

lque la sangre ! la nicre jumtas le recuerdan el fresco color que senia la faz de su amiga.]

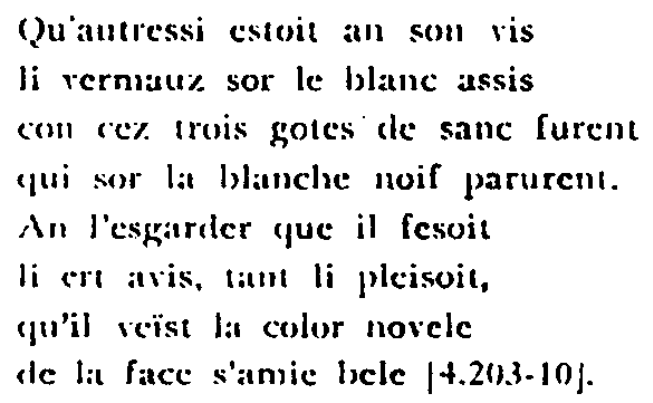

[Porque del mismo modo esiabal en su rostro lo rojo puesto sobre lo blanco como se hallaban ayuellas tres gotals de sangre que aparecicron sobre la bianca nicre. Mientras lo comemplabal le guslaba tanto que le parecia que reía el color joven de la faz de su hermosa amiga.]

Wolfram von lischenbach tradujo bien la idea de Chrétien en el primer pasaje de los dos que antes se han transcrito, pero la desfiguró totalmente en el segundo, cuando dice yue las tres gotas de sangre cstaban dispuestas de tal modo que dos de ellas recorraban a Parzival las incjillas de Cundwiramurs y la otra su mentón. Si nos atcnemos a la idea de Chrétien. mucho mayor es aún li: incomprension que revela el autor del pereder, el cual. cridentemente influirlo por el motio folklórico, estropea lat belleza del simil de Li conles del Graal al disgregar y aumentar los elementos coloristas: la negrura del cuervo (fue sugiere la cabelleria, la blancura de la nieve que sugiere ha piel $y$ la roje\% de la sangre que sugiere las mejillas. De esta sucrte. el simil pierde su sutileza y su clegancia y vuclve al primitivismo, que tiene el motivo fol. 
klórico en algunas versiones, entre ellas la de $E l$ destierro de los hijos de Usnech. En el Peredur se ha hecho vilain to que en Li contes del Graal era courtois.

El símil de Chrétien de Troyes se pucde asediar desde un punto de partida muy distinto. Cuando presenta a Blancheflor en el castillo de Belrepcire, Chrétien acumula los consabidos tópicos de la descriptio pucllac y retrata el tinte de su rostro con las siguientes palabras:

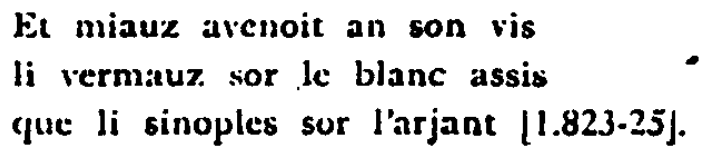

IY mejor sentabat en su rostro lo rojo sobre lo blanco que el sinople sobre la plata.]

El símil heráldico es muy adecuado a una novela de este género, y obsérvese que en francés antiguo, y concretamente en Li contes del Graal (cf. verso 1.221), sintoples es exactamente el color rojo. Esta primera indicación sobre el rostro de Blancheflor anuncia el episodio de las gotas de sangre sobre la nieve, y tanto es así que, en éste, Chrétien repetirá literalmente el segundo de los versos ahora citados :

Qu'autresi cstoit an son vis li vermauz sor le blanc assis con cez trois gotes de sanc furent... [4.203-05].

Para indicar que los colores rojo y blanco se mezclan y funden y dan el sonrosado del rostro, Chrétien emplea el verbo anluminer. Perceval explica su contemplación a Gauvain con las siguientes palabras:

Que derant moi an icest leu aroit trois gotes de fres sanc, qui anluminoicnt le blanc. 
An l'esgarder m'estoit avis

que la fresche color del vis

m'anic la bele i veïsse $[4.450-55]$.

IAnte mi en este lugar habia tres gotas de sangre fresca que ilu. minaban el blanco. Al inirarlas me parecia que vicse cl fresco color del rostro de mi amiga la hermosa.]

En el mismo Li conles del Graal, Chréticn, al describir a la hermana de Gaurain, dice:

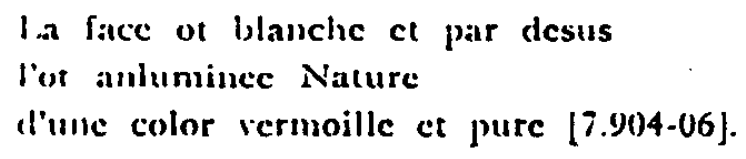

[tal far tenia blanca : Naturaleza la había iluminado por encima (the un color rojo y puro.j

En terminos similares habia hablarlo Chrétion de la protagonista de su primera novela artúrica, Erec et Enide:

Plus of que n'est la flors de lis cler et bianc le front et le vis;

sor la color. per grant mervoille, d'unc fresche color vernoille, que Nature li ot donec, estoit sa face anlumince $[427-32]$.

[Más que lo es la llor del liries tenia la frente y el rostro claroy blanco; por gran maravilla sobre el color estaba su faz iluminada por un fresco color rojo que Naturaicza le halıia dado.]

También en el Cligés, al describir la belleza de Soredamor, Chrétien había insistido en el

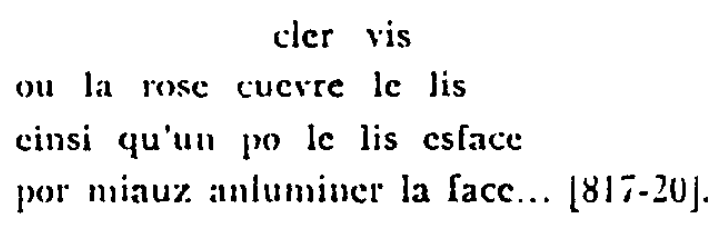

[claro rostro donde la rosa cubre al lirio de tal modo que el lirio quecla un poco borrado para mejor iluminar la faz.. 
listos ejemplos nos acaban de precisar el sentido del sinil de las gotas de sangre en la nieve. lis decir: lo esen. cial no son los elementos sangre y nieve separados (como. ocurre en El deslierro de los hijos de Usuech, en el Peredur y en un momento desdichado de Wolfram von Eschenbach), sino la blancura (del lirio o de la nieve) iluminada. por la rojez (de la rosa o de la sangre). Ir esto tiene una: vicja y noble tradición literaria.

Cuando Lavinia oye a su madre mentar el nombre de. Eneas. la joven se ruboriza, y Virgilio compara el cambio de color del rostro con el marfil, que picrde su blancura a causa de la sangrienta púrpura, o a los lirios, quecurojecen si se les mezcla con abundantes rosas:

Indum sanguineo ueluti uiolaucrit ostro

si quis ebur, aut mixta rubent ubi lilia multa

alba rosa: talis uirgo clabat ore colores [Alen., XII, 67-69].

Entre la gran diversidad de términos de color que apa-recen en la poesia latina clásica nos importa ahora destacar la frecuencia con que sanguinems indica el color rojo. oscuro. hasta el punto de llegar a hacerse sinónimo de ruber y puniceus ${ }^{3 "}$, y lo gencralizado que cstá niueus como. sinónimo de albus y candidus, sobre todo en descripciones del cuerpo humano ${ }^{33}$. Frecuente es también en -los. poetas latinos, que la heredaron de los alejandrinos, la comparación. tan natural, de niue candidior (por ejem. plo, aplicada a caballos blancos, en Virgilio, Aen., III,. 537-S, y en Ovidio, M/\%., VIII, 373-4) ${ }^{34}$. La mezcla de $n i-$ ueus con lo rojo (purpurcus, rubor, roseus) se convierte.

32 Véasc J. ANmkt, Eitude sur les termes de couleur dans la lansue latine, Paris, 1949, paig. 113.

3.3 Mid., pigs. 39-40 y.325.

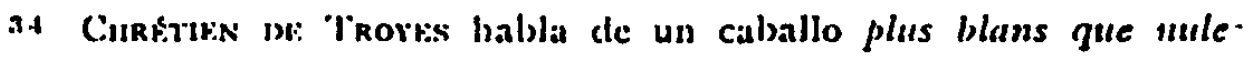
mois (Clisers, verso t.(1.36). 


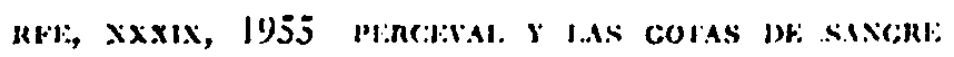

en un recurso frectente para describir el tinte del rostro. Tibulo, al pintar al dios del Amor, dice:

Catuclor etan quatem pracfert Latonia l.una, et color in niuco corpore purpurens [III, 4, 29-34].

Abundan los ejemplos en. Oridio:

... et in ninco mixtum candore ruborem [.Met., III, 42.3].

Quique subest niuco lenis in ore rubor [Her., $\mathrm{xx}, 120]$.

Candicla candorem rosco suffusa rubore

ante stetit: niuco lucet in ore rubor Amores, 111, 3. 5-6j.

Estacio describe a Aquiles cuando regresa de combatir:

dukis adhuc uisu, niuco natat ignis in ore purpurcus [Ach.. I, 161-2].

El contraste entre la nicve y la sangre aparece en ef Canto funclbre a Adonis, de Bión, cuando el pocta dice que "sangre negra se clerrama sobre su carne de nieve":

...

.

Concepto que se repite. casi literalnnente. en la descripción de la muerte de un guerrero por Estacio:

Ibat purpurcus niuco de pectore sanguis [The., IX, $8 S 3$ ].

Este contraste de colores, tan frecuente en la poesia latina clásica, fué recogido por los autores de las artes poéticas medievales, que lo incorporaron a sus tratarlos, y dicron así cicrta obligatoriedad a la idea en los cánones de la descriptio superficialis. Ein el cjemplo de descripción del rostro que da la Poctria nowa, de Geolfroy: de Vinsaut, no latta el cletalle: 
Aemula sit facies Aurorac, nec rubicundac nec nitidac, sed utroque simul neutroque colore 35 .

Y Matthicu de Vendôme ejeinplariza en su Ars versificatoria:

$$
\begin{aligned}
& \text { Cimdori socio rubor interfusus in ore } \\
& \text { militit, a roseo fore tributa patens } 36 .
\end{aligned}
$$

El simil con la nieve y la rosa sigue manteniendo su vitalidad en la poesía latina medieval. Fin la comedia elegíaca Alde, de Guillaume de Blois, se dice que el rostro de la doncella supera en blancura a las nieves y en tinte a las rosas, pues en dil la rosa colorea el lirio y en sus mejillas se mezclan el ardor de la purpura y el candor de la nieve:

Alba caro niuibus similişue rosis color esset, si non illa niues uincerel, ille rosas.

Virginis in facie rosa lillia pingit, et ardet albetque in tencris purpura nixque genis $[127-130]^{3 i}$.

En la lírica provenzal es harto frecuente comparar la blancura de la piel femenina con la de la nieve. Bernart de Ventadorn, contemporáneo de Chrétien de Troyes, escribe:

$$
\begin{aligned}
& \text { Qui ve sas belas faissos, } \\
& \text { ab que m'a vas se atraih, } \\
& \text { pot be saber atrazaih } \\
& \text { que sus cors es bels e bos } \\
& \text { c blancs sorz la vesticlura } \\
& \text {-cu non o dic mas per cuda-, }
\end{aligned}
$$

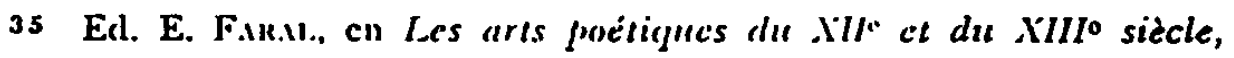
Paris, 1923, paig. 214.

36 Ibid., paig. 129.

3: Ed. M. WINtawrithtise en LA "Commédie" latine en France au XII" siècle, textes publices sous la direction... de G. Cones, 1, París, 1931, prifg. 1.35. 
que la neus, call ilh cs nuila, par vas lei brun'ct cscura 3 .

|Aquel que ve sus hermosas facciones, con las cuales me ha atrat(lo) hacia sí, puede bien saber al punto que su cucrpo cs bello y bueno Y blanco bajo las vestiduras - lo cligo por sola suposición-, pues la nieve, cuando clla csla clesnuda, es a su lado morcna y uscura.]

$$
\begin{aligned}
& \text { cors blane tot atretal } \\
& \text { comi lat neus a madal s!. }
\end{aligned}
$$

[cuerpo banco, igual que la nieve en Navidatl.]

Armaut de Maruclh hablará de Menlon e gola e peitrina Blanca coml la neus ni flors d'espina" ", y muchos serán los trovadores yue recurran a esta tan obvia comparación. l'cro muchos son también los gue scinalan el contraste cntre la nicve y la rosa para describir el tinte de la piel o de un rostro. En una canción atribuída a Aimeric de Belenoi se pondera:

lit col tos temps hom non poiria dir lat gran beutat, ni escriur'en uns breus, del seu cors clar pus qe ro\%at ni neus 1 .

[No habria licmpo suficiene para decir su gran belle\%a ni para describir en un mensiljc su cucrpo, más claro que rosa y nicve.]

Peire Vidal cxclama:

Bonal clomna, neus de port scmbla la rostra blancors,

38 Canción A tantas bohas (A. PILiET Y H. CARstess, Bibliographie der Troubadours, Hallc, 1933, 70, 8), versos 3j-th; ed. C. Appel, Bernart ion Ventadorn, Halic. 1915, paig. 52.

39 Lo gens tems (Phitet-Cakstress, 70, 28), versos 37-38; ed. Appril, prig. 167.

10 Domna genser (Pinlti:-CARTKks, 30, III), ictsos 97-98; ed. M. DE: Riquer, Ia lirica de los trovadores, I, liarcelona, 1948, pág. 474.

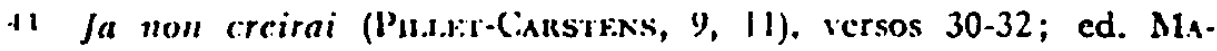
nî Dumrrnescu, Poésie's du troubadour dimeric de Belenoi, Paris, 19.35, pisg. I+1. 


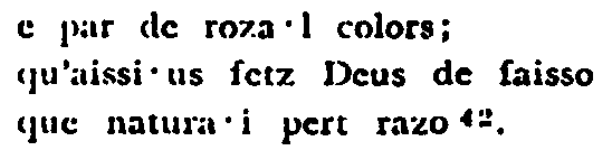

[Señora: nieve de montaña semeja vuestra blancura y el color parece de rosa; porque Dios os hizo de sucrte que la Naturaleza pierde sus derechos.]

in el roman provenzal de Jaufré, al describirse la belleza de Brunissen, lecmos:

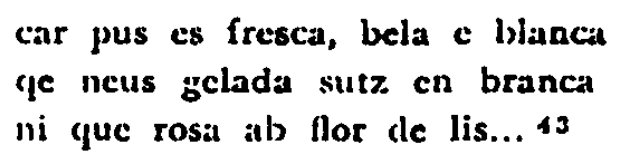

\{pues es mas fresca, bella y blanca que niere helacla encima de th rama y que rosa con lirio.]

Con refinada elegancia, el trovador Folquet de Marselha se vale del contraste entre la blancura y el colorado para solicitar a su dama. que tan bien sabe conciliarlos, que ponga de acuerdo al Anor con la Piedad:

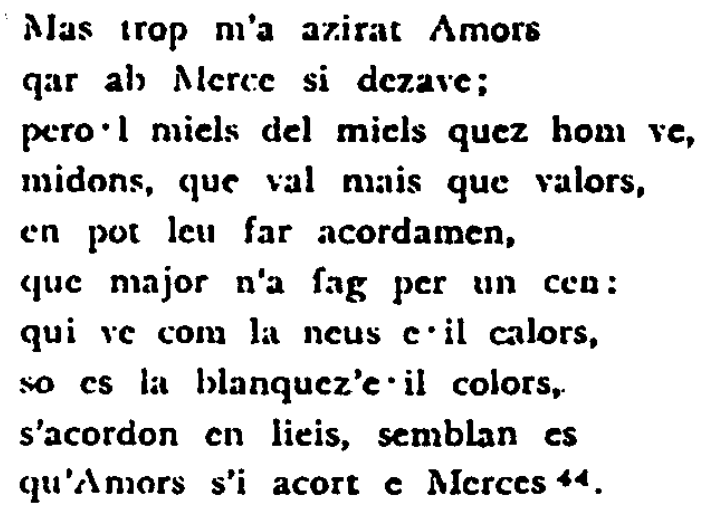

[Alucho me ha odiado el Amor al desarenirse con la Piedad; no

12 Atressi col perilhans (Pliky-Caktrins, 364, 6), versos 56-60; c:i J. Anglibe, les poesies de Peire Vidal, Paris, 1913, pig. 5.

4 Ed. C. Bkusil., Jaufré, roman arthurien du XIII0 siécle eł iers provençaux, 1, París. 194.3, pág. 110, versos 3.141-4.3.

it Mout $i$ fetz ('miliet-Cakstens, 155, 14), versos 31-40; ed. S. Stroosski, Le troubadour Folquet de Marscille, Cracovia, 1910, pafinal +2 . 
olistante mi señora, que vale más que el propio valor, puecie conciliar faicilmente la mejor de cuantas cosas bucnas se ven, pues ha hecho algo que vale cien veces mis: cuando uno ve que la nieve y el calor, esto es, lo blanco y lo colorido, sc concilian en clla, parece que del mismo modo en elia se conciliarian el Amor y la Piedad.]

Aquí el trovador, al tomar como comparación de su estado de ánimo (amor no correspondido) el contraste entre la nieve y lo colorado del tinte de la dama, pone bien de manifiesto que esta mezcla de colores es cosa perfectamente conocida de su auditorio. Y el trovador Sordel (el italiano Sordello). que en unit canción repite que el color de su dama par flos ab neu quan chait", cn otra alesarrolla ampliamente el contraste en su estrofa inicial:
Atret:u dei ben chantar finamen
d'isern com far. d'estiu, segon rason, per c'ab lo freitz voill far gaia cunson que s'en pascor de chantar cor mi pren, quar la rosal sembla lei de cui chan, aultresi es la neus del sieu senbliun: per qu'en andos dei per s'amor chantar, tant fort mi fan la rosa $c \cdot 1$ neus menbrar 40 .

LFs razonable que cante tan sinceramente en invierno cono lo hago en verano; así, pucs, con el frío quicro lacer una canción aleyre icl mismo modo que en primavera siento ganas de cantar; puez lat rosa se parece a aquella de quien canto y asi es la nieve como su semblante: por lo que en ambas [estaciones] debo cantar por iu amor, tanto ine la recuerdan la rosia y la nicre.]

Estc último ejemplo es importante en nuestra indagación, porque tanto Perceval como Sordel recuerdan la belleza de la dama por la sugestión producida por los elementos de la naturaleza, punto que vamos a considerar inmediatamente.

t.5 Tos lemps serai (P'lli.er-Carstens, 437, 36), versos 37-38; ed. M. Boni, Sordello, Le poesic, Bolonia, 1954, paig. 68.

16 Atretan dei (Puller-Carstixs, 437, 5), versos. 1.8; cd. Bon, jaigina 15. 
En los origenes del ensimismamiento de Perceval ante las tres gotas de sangre en la nieve está, evidentemente, el motivo folklórico, que Chrítien de Troyes pudo hallar en su propio ambiente, en su nativa Champagne, sin ne. cesidad de recurrir a tradiciones exóticas. No obstante, el motivo folklórico suele presentar los elementos coloristas disgregados $c$ imagina un ser con la picl blanca como la nieve, las mejillas rojas como la sangre y el cabello negro como el cuervo, tal como aparece en El destierro de: los hijos de Usnech y en el Percelur. Pero Chrétien de Troyes conoce perfectamente el motivo clásico, tan frecuente en Ovidio -autor que tradujo en su juventud e. imito en toda su vida de escrjtor ${ }^{17}$ - que destaca la mezclat de lo niuceus con lo rojo, contriste que tendrá pervivencia en la lírica medieval. que será aconscjado en las: artes pociticas y. que recogerán los escritores en vulgar. Lo esencial del motivo culto es la mezcla de ambos colores: in niuco $\mathrm{mix}$ tum candore ruborem. En Li contes del Graal se da la feliz convergencia del tema folklórico con c! tema clásico, y ello nos hace comprender la verdadera razón de que Chrétien haya prescindido del elemento. cuervo-caliellera negra que aparece en el primero. No prescindió de él únicamente porque Blancheflor fucra rubia, pues Chrétien no riene una manifiesta antipatía por las. morenas. y Lunete es una avenanz brunele (Li chevaliers aII lion. verșo 2.+16). sino porcque el elemento negro- le. impedia que las tres gotas de sangre iluminuran la blan. cura de la nieve, con lo queda patente que lo que busca es la mezcla de ambos elementos. Lo corroboran algunos. seguidores $\mathrm{c}$ imitadores de Chrétien. En Fergus ct Galienc. se lee que la Naruraleza

it Viase F. E. Gurin, The influence of Ovide on Chrestien de. Trojes, The Romanic Review, XII, 1921, págs. 97-134 y 216-247, y

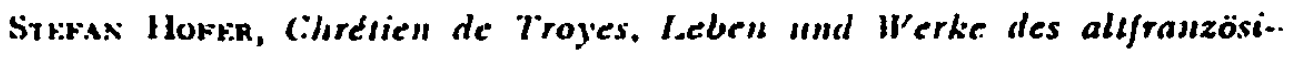
schen Epikers, Graz-Kïln, 1954, piigs. 50-58. 


$$
\begin{aligned}
& \text { lor le blancor enluminier } \\
& \text { i mist une color vermelle } \\
& \text { lele cu'cl mont n'ot sa parclle. } \\
& \text { Quiml Nature ot ens en son vis } \\
& \text { le rermel sor le blane asis, } \\
& \text { molt par li plot a regarder } 4 \text {. }
\end{aligned}
$$

Ilara iluminar la blancura puso un color bermejo, tal que no tc. nia par en el munde. Cuando Naturalcza hubo colocado en su rostro. ln bermejo solure in blinco, le plugo extraordinariamente contemplarko.]

bin el Galeran de Brelagne la idea se precisa tal rez aún más :

bi lo vis blan com fleur de lis, destrempe de couleur vermeille, a qui rose ne s'aparcille $[1.282-4] 19$.

IY el rostro blanco conno fior de lirio. empapado de color berme.jo. que ni con la rosia pucde compararse.]

Como en el tema folklórico. en el episodio de Li conles del Graal la imagen aparece invertida. Lo inmediato. y lo clásico es aue lo sonrosado del rostro de una mujer sugiera la mezcla de nieve con sangre: en Chrétien. 'a. nieve iluminuela por la sangre es lo que sugiere a Perceval el color del rostro de Blancheflor. El término real de lat imagen ha pasator a ser el término irreal. y vicerersa: se trata. pues. de una inagen reversible". P'ero para llegar at esta inversión de la imagen ni tan sólo cra preciso.

ix Tomo lat cital de unit nota de $A$. Hex.s, en Der Percesalroman, prigina 658.

19. Jean Rraste, (;aleran de Brelagne, ed. L. Fond: "I cs classiques français d" Moyen Age". París, 1925, prig. t). El pasaje del' Cristal et Clarie, que reproduce $A$. Hilks en una nota (Der Percezalroman, prig. 707), es una eviclente imitación de Chrétien. Yéase: Nalure qui del faire ert sage, Ot asanble par mariage $E_{t}$ asis le vermeil ol blanc, Bel altresi con sor moif sanc.

$\therefore$ Sobre la imagen reversible, véase Dimaso Arosso, Eistudios $y^{\text {- }}$ ensayos gongorinos, Naclrid, 1955, paigs. 48-49, "Biblioteca Rominica, llispánica". 
el motivo folklórico: bastaba con que la imagen, en su .sentido recto, estuviera muy divulgada, casi gastada; y ya hemos visto que el trovaclor Sordel llega a la misma inversión sin qque tenga para nada en cuenta el motivo folklórico y sin recordar en aquel momento $\mathrm{Li}$ contes del -Graal.

Chrétien de 'T́royes preparú el cpisodio que aquí he'mos cstudiado con la intención puesta en la imagen poética. Nos lo demuestra el hecho de que nuestro episodio tiene lugar unos días después de Pentecustés, o sea, cn tiempo en que en la laás de Gales no suele nevar. Los criticos han llamado la atención sobre esta incongruencia ${ }^{62}$, pero ya antes se dió cuenta de ello Wolfram von Eschenibach y disculpó a Chrition con gracia y acierto:

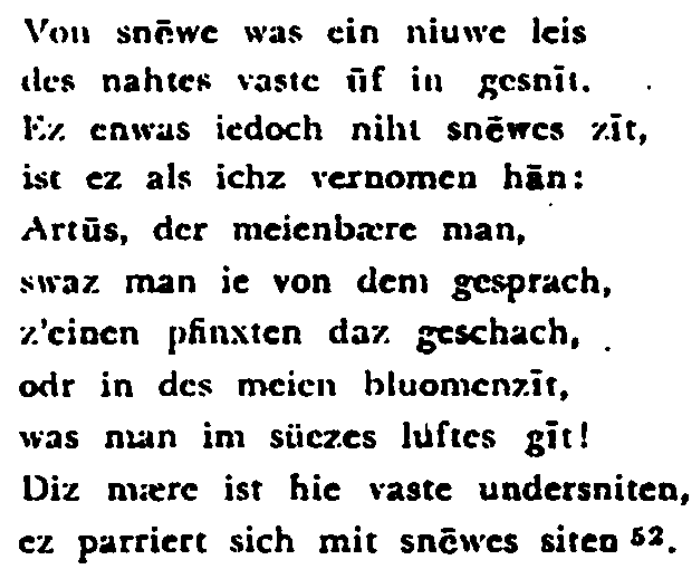

[Durant la nuit une fraiche et abondante couche de neige était :tombec sur lui. Ce n’était pourtant pas, si j'en crols l'histoire, le temps des neiges. Car toutes les aventures qu'on nous rapporte d'Ar'tur, héros printamier, se déroulent à le Pentecôte ou durant le beau mois fleuri de mai. Comme les poetes aiment à l'entourer de brises doucement parfumées! Mais ici l'histoire nous presente des teintes contrastées: on $y$ voit à lit fois des fleurs et de la neigel ${ }^{5.3}$.

il Cfr. R. S. l oonts, Arthurian tradition, píg. +15 , y J. Frappiek, I.: roman breton: Chrétien de Troyes, Perceval ou le conte du Graal, jaigina 68.

$\therefore=281,12-22$, texto dic A. Mlokt:a, paig. 126.

it lrad. 'l'osstist, I, paig. 24.5. 
La intempestiva nevada puede romper la verosimilitud climatológica de $l i$ contes del Graal, pero era necesiruia, desde el punto de vista pótico. para sugerir a Perecval los colores de la far de Blancheflor.

MakTía de RiQuek 